\title{
Early Restenosis of Sirolimus-eluting Stent: An Unusual Case of a Hyperthyroid Patient
}

\author{
Syeda Javeria Shabbir ${ }^{1}$, Mariam Baloch ${ }^{2}$, Faryal Mustafa ${ }^{1}$, Hira Maab $^{1}$, Laila Tul Qadar ${ }^{1}$ \\ 1. Internal Medicine, Dow University of Health Sciences, Karachi, PAK 2. Medicine, Dow Medical College, Dow \\ University of Health Sciences, Karachi, PAK
}

Corresponding author: Hira Maab, hiramaab@gmail.com

\begin{abstract}
The presentation of atherosclerosis with concomitant hyperthyroidism is not uncommon. Hyperthyroidism predisposes to worse cardiovascular pathologies like systolic hypertension, atrial fibrillation, and hypercoagulability. Drug-eluting stents, on the other hand, have emerged as a miracle treatment choice for patients having atherogenic conditions. They have the highest success rates when it comes to minimizing in-stent restenosis (ISR) during short-term follow-up. There is scarce literature that assesses the correlation of multinodular goiter (MNG) to ISR, especially in Pakistan, and thus any probable association between the two is left untouched. We report a case of a 57 -year-old female who is a known hyperthyroid with a massive MNG, presenting with worsening chest pain. She had undergone sirolimus-eluting stent (SES) implantation in left anterior descending artery (LAD) six months back. Cardiac catheterization confirmed restenosis of the SES in the LAD, along with the occlusion of left circumflex and right coronary artery, accompanied by grade I diastolic dysfunction and mild aortic regurgitation on echocardiography.
\end{abstract}

Received 12/24/2019 Review began 12/25/2019 Review ended 12/26/2019 Published 12/28/2019

๑) Copyright 2019 Shabbir et al. This is an open access article distributed under the terms of the Creative Commons Attribution License CC-BY 3.0., which permits unrestricted use, distribution, and reproduction in any medium, provided the original author and source are credited.
Categories: Cardiac/Thoracic/Vascular Surgery, Endocrinology/Diabetes/Metabolism, Internal Medicine Keywords: hyperthyroidism, drug-eluting stents, multinodular goiter, cardiac catheterization, coronary stents, stent thrombosis, in-stent restenosis

\section{Introduction}

Hyperthyroidism is defined as excessive T3 production due to thyroid gland hyperfunctioning. Peripheral monodeiodination of $\mathrm{T} 4$ is increased, which ultimately results in reflective changes in the cardiovascular system [1]. A fraction of patients having thyroid disease have cardiac disease, either coronary or valvular, as a common finding [2]. In exceptional cases, hyperthyroid patients can present with chest pain and echocardiography changes of cardiac ischemia [3]. In elderly patients with coronary artery disease (CAD), an increase in cardiac contractility and workload associated with hyperthyroidism reveals an increase in myocardial oxygen demand [1]. There is a higher risk of CAD and increased death rate associated with overt and subclinical hyperthyroidism [4]. Hyperthyroidism has been related to an increased activity of factor X in coagulation cascade which is simultaneously related to increased risk of CAD [5]. Restenosis is defined as a decrease in the luminal diameter of more than $50 \%$ after percutaneous coronary intervention, either with or without stent implantation. Interventional cardiologists have always considered in-stent restenosis (ISR) to be the main culprit, and hence many techniques have been introduced in the last 20 years to reduce its incidence: first newer generation bare-metal stents (BMS), then drug-eluting stents (DES), and finally drugcoated balloons [6]. With the arrival of DES the incidence of restenosis has reduced to numbers $<10 \%$ [7]. Here, we present the case of 57-year-old female, a known hyperthyroid, brought to the hospital with acute chest pain which was later established as secondary to the restenosis of sirolimus-eluting stent (SES) in the left anterior descending artery (LAD).

\section{Case Presentation}

A 57-year-old female patient presented with gradual and progressive chest pain (Canadian Cardiovascular Society Grade III). Her past medical history included diabetes mellitus, hypertension, and hyperthyroidism. Previous surgical procedures included angioplasty where sirolimus-coated DES of $2.5 \times 27$ and $2.5 \times 19$ was placed in the LAD six months back. She had been taking neomercazole $5 \mathrm{mg}$ for hyperthyroidism, glimepiride $2 \mathrm{mg}$ for diabetes, and $160 \mathrm{mg}$ valsartan for hypertension. On inspection, a prominent goiter was seen on her neck that moved with swallowing along with a median sternotomy scar, which indicated her previous thoracic surgery (Figure 1). 


\section{Cureus}

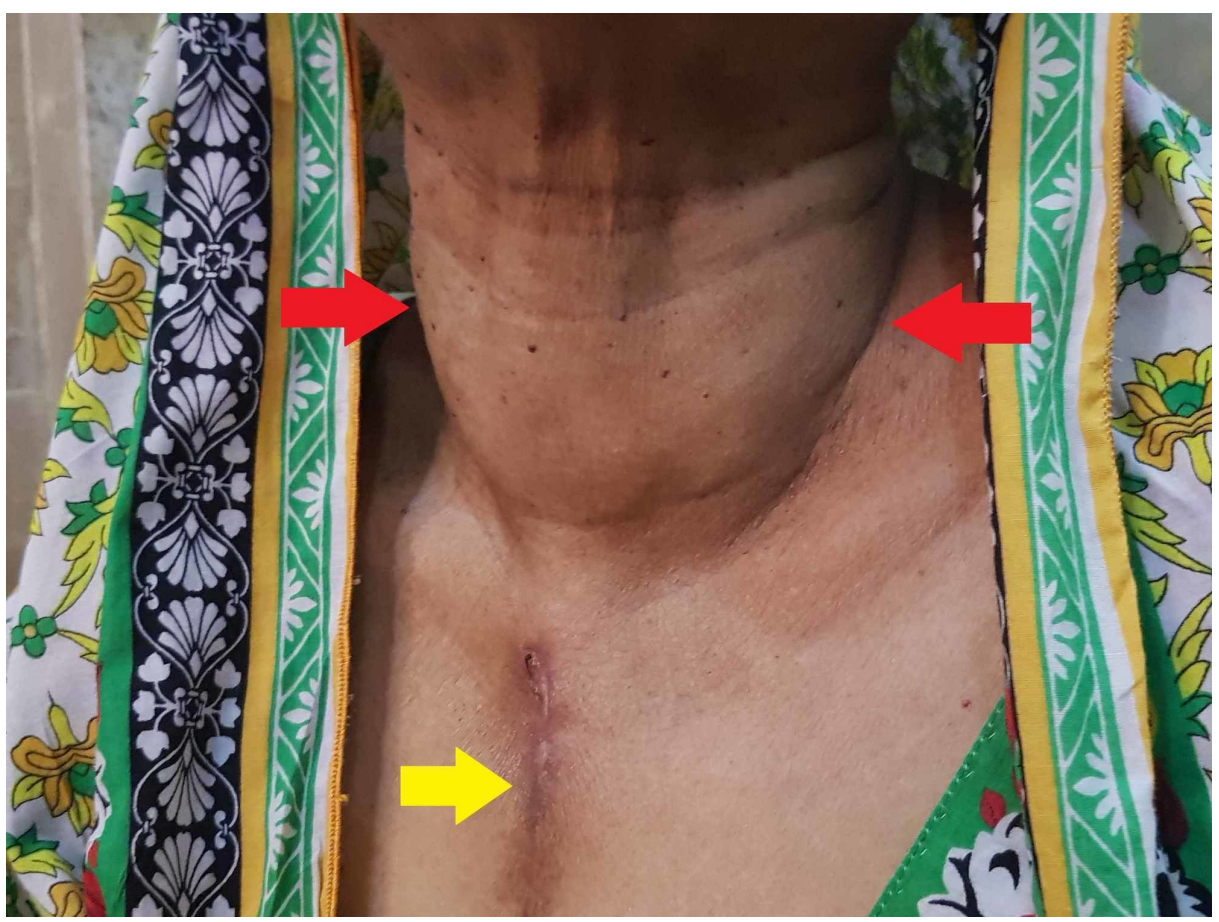

FIGURE 1: Visible goiter (red arrows) along with a clear previous median sternotomy incision scar (yellow arrow)

On admission, the patient underwent cardiac catheterization which revealed the following findings: left main stem was normal, left circumflex artery was $90 \%$ occluded from proximal to mid with $80 \%$ disease in obtuse marginal 1 . Right coronary artery was also diffusely diseased with $70 \%-80 \%$ mid vessel disease (Figure $2 A$ ). LAD also showed $40 \%-50 \%$ proximal diffuse stenosis, followed by ectatic segment with ISR in the mid to distal LAD-ectactic segment (Figure $2 B$ ).

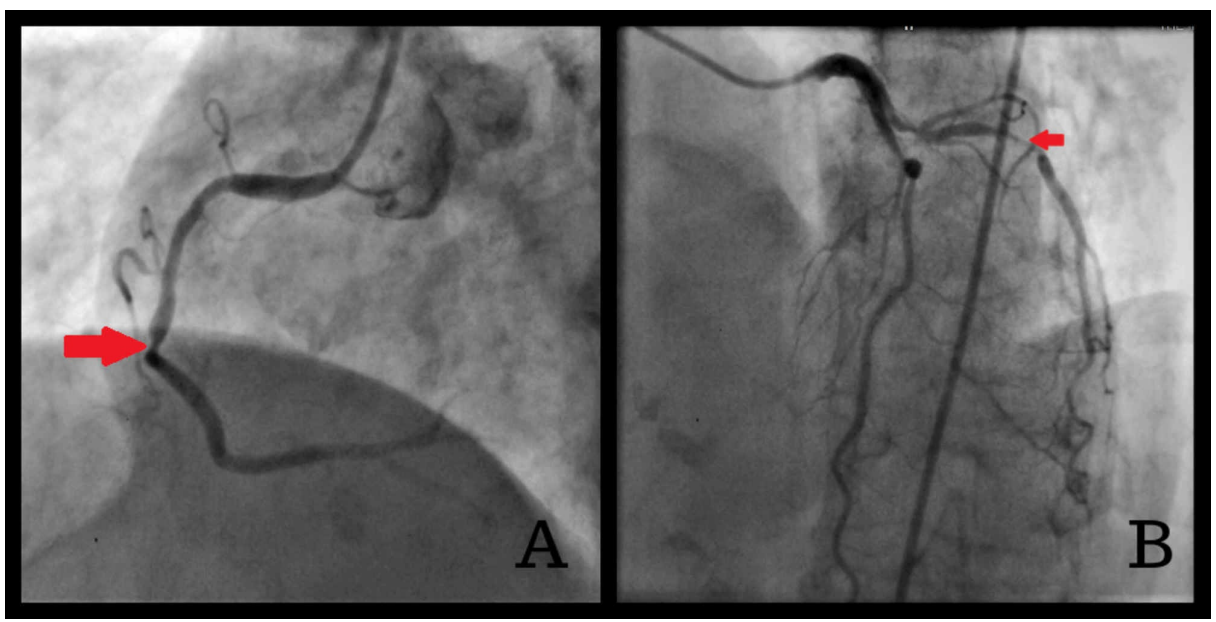

FIGURE 2: Coronary CT angiography images on admission (A) marked occlusion of mid-RCA (B) in-stent restenosis of the LAD

$\mathrm{CT}$, computed tomography; RCA, right coronary artery; LAD, left anterior descending artery.

Moreover, her Echo/Doppler cardiography report revealed grade I diastolic dysfunction and mild aortic regurgitation with normal left ventricular size and systolic function. Cardiac investigations were followed by thyroid profile where slightly low thyroid-stimulating hormone level was seen; however, T4 and T3 levels were normal. This was because she was kept on medication with adjustment made in dosages to achieve euthyroid levels. Later upon subsequent ultrasound of neck, multinodular goiter in the right side of $3.2 \times 2.4$ $\mathrm{cm}$ (Figure 3) and in the left side $0.4 \times 0.2 \mathrm{~cm}$ (Figure 4) was observed. 


\section{Cureus}

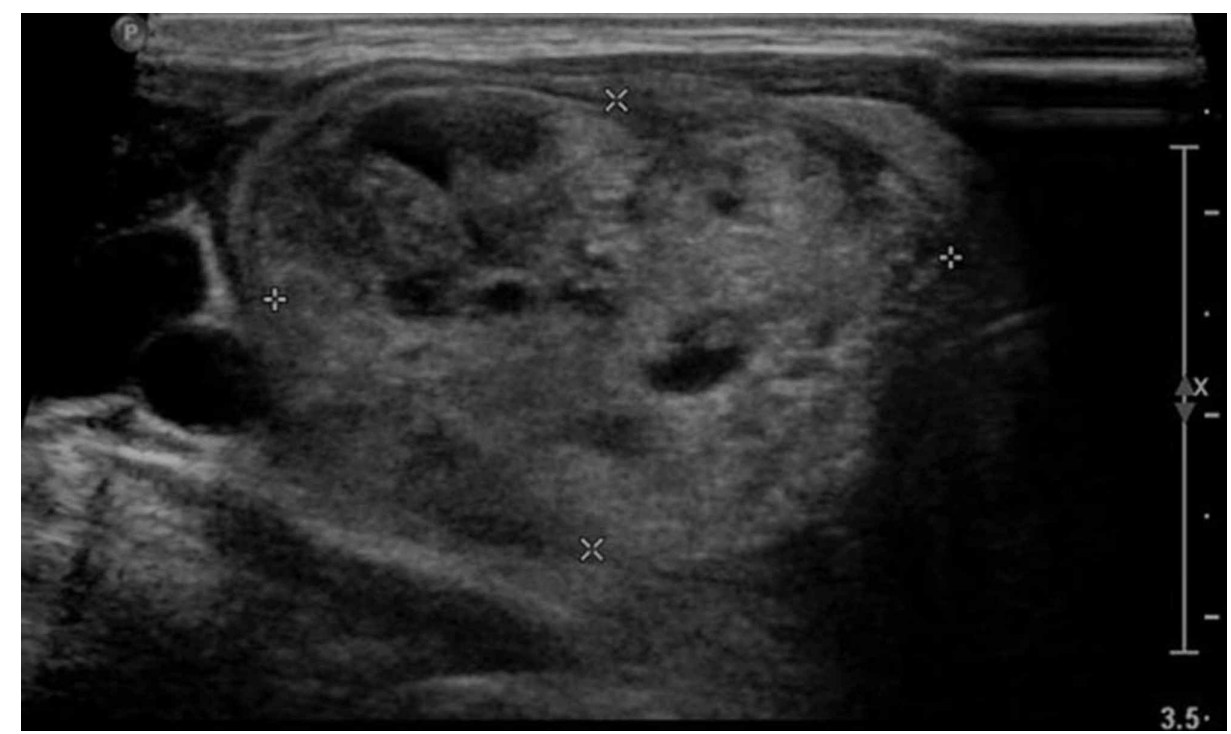

FIGURE 3: U/S of neck multinodular goiter in the right side measuring

\section{$3.2 \times 2.4 \mathrm{~cm}$}

$\mathrm{U} / \mathrm{S}$, ultrasound.

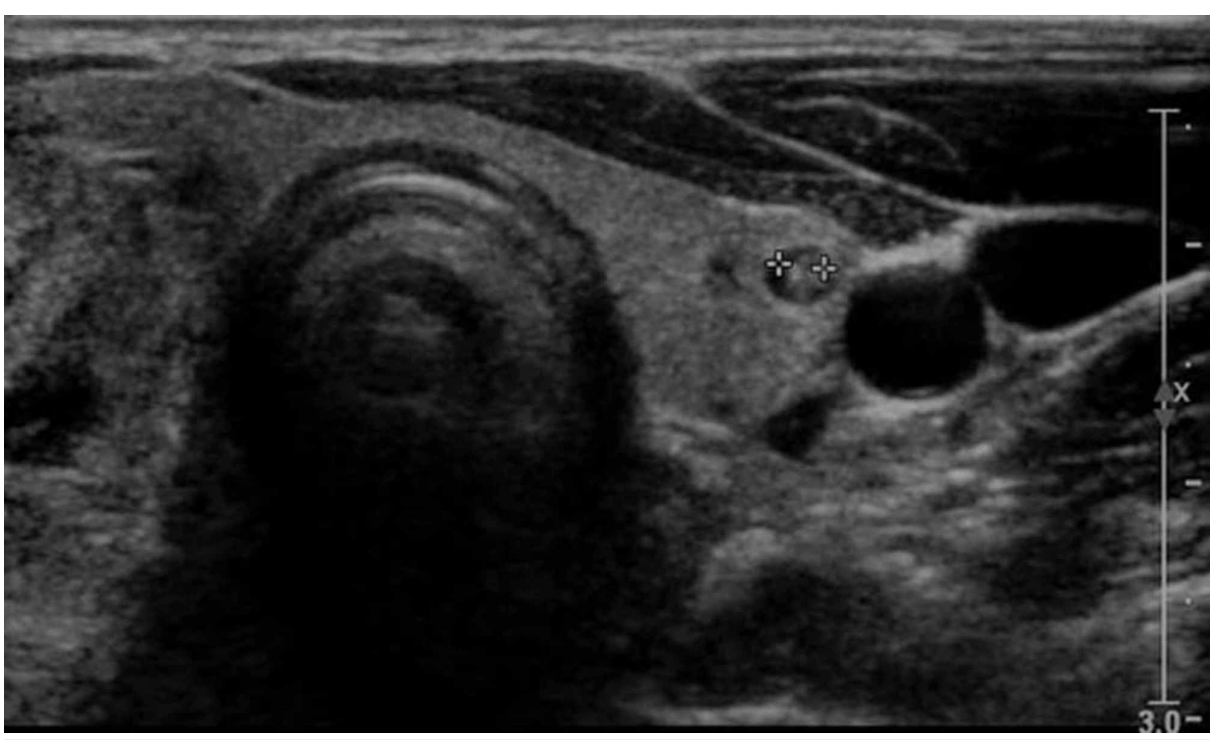

FIGURE 4: U/S of neck multinodular goiter in left side measuring $0.4 x$

\section{$0.2 \mathrm{~cm}$}

$\mathrm{U} / \mathrm{S}$, ultrasound.

The patient was prepared for coronary artery bypass grafting $(\mathrm{CABG})$ with pre-operative assessment showing a euthyroid state. After surgery, she was shifted to intensive care unit and was discharged a week with no post-operative complications. A follow-up of six months was recommended to assess the patient condition.

\section{Discussion}

From BMS to the development of the second-generation DES, coronary stents have undergone a remarkable transformation with the aim to avoid both restenosis and thrombosis. The risk, however, is still present attributed to the stent being perceived as foreign prosthesis and subsequent neointima proliferation as the likely underlying pathology [8]. Variable investigations have looked into the predictors of ISR and recognized small vessel size, stents of increased length, diabetes mellitus, history of bypass surgery, and complex looking lesion as some of the metrics [9] 
Although DES have been in Pakistan since 2002, few pieces of research have looked into the phenomenon and have been mostly centered around possible interventions like drug-eluting balloon and bioresorbable vascular scaffolds in the wake of ISR [10]. To the best of our knowledge, only a case of a late ISR was reported in October 2005 in the Pakistani literature and just a single investigation listing hypertension, diabetes mellitus, positive family history, dyslipidemia, and smoking as some of the triggering factors [11]. Even though the presentation of thyroid dysfunction as multinodular goiter is not that uncommon, the coexistence of multinodular goiter and its influence on the sequelae of coronary heart disease have never been emphasized in prior publications in Pakistan. Our case is, therefore, distinctive drawing attention to the comorbidity of multinodular goiter in ISR patients in the local population and the role played by the clinical parameter of hyperthyroidism on the outcome of the percutaneous intervention. To this effect, computed tomography angiography findings of patients with overt and subclinical hyperthyroidism had revealed a greater extent of high-grade and overall coronary stenoses, plaque burden, and high-risk plaque hallmarks than the euthyroid patients [12]. The data from Canpolat et al. substantiate this point, whereby the preprocedural serum level of fT4 was found to be a significant independent factor of ISR in patients with successful BMS implants [13].

Data on the overall epidemiology of MNG came up vague on our literature search; however, a study on histopathological findings of 624 thyroidectomies in Holy Family Hospital, Rawalpindi, Pakistan, did corroborate that MNG is the most common non-neoplastic lesion, with presenting age of patients ranging from 10 to 90 years. This emphasizes the importance of its cardiovascular implications, particularly in older patients who also have age as a non-modifiable risk factor [14].

ISR, on its own, is a nightmare for interventional cardiologists, with ongoing measures and precaution to circumvent this complication. With concurrent MNG and hyperthyroidism, it, without saying, puts the patient under a greater risk of ISR with high relevance in routine clinical practice. A close follow-up in such patients is mandatory. Our patient after primary percutaneous coronary intervention was lost to follow-up for six months after which she later presented with not only ISR but also the involvement of left circumflex and right coronary artery, thus progressing to three-vessel CAD with surgical revascularization through $\mathrm{CABG}$ as a reasonable option.

Here we want to highlight that while sifting literature we came across case reports where concomitant thyroidectomy was coupled with CABG with improved post-surgical outcomes, in addition to saving the patient from a second surgical intervention [15]. That being said, this was not a plausible option in our set up with its limited resources. In any event, this multidisciplinary approach should be kept in mind and given due consideration should the technical expertise and resources are available for surgical execution.

\section{Conclusions}

Herein, we report a case of ISR in a known hyperthyroid patient. It is crucial to know any associations of altered thyroid function in the development of stent failures, be it in the form of ISR or stent thrombosis. Studies evaluating this relationship are meagre. Moreover, stent failure presents as a medical emergency; thus, it is essential for the cardiologists to know the triggers which cause it so as to maximize the efficacy of these tubes.

\section{Additional Information \\ Disclosures}

Human subjects: Consent was obtained by all participants in this study. Conflicts of interest: In compliance with the ICMJE uniform disclosure form, all authors declare the following: Payment/services info: All authors have declared that no financial support was received from any organization for the submitted work. Financial relationships: All authors have declared that they have no financial relationships at present or within the previous three years with any organizations that might have an interest in the submitted work. Other relationships: All authors have declared that there are no other relationships or activities that could appear to have influenced the submitted work.

\section{References}

1. Klein I, Danzi S : Thyroid disease and the heart. Curr Probl Cardiol. 2016, 41:65-92. 10.1016/j.cpcardiol.2015.04.002

2. Ranasinghe AM, Bonser RS: Thyroid hormone in cardiac surgery. Vascul Pharmacol. 2010, 52:131-137. 10.1016/j.vph.2009.11.004

3. Iiya M, Yamawake N, Nishizaki M, et al.: Ventricular fibrillation induced by coronary vasospasm in a patient with early repolarization and hyperthyroidism. Intern Med. 2018, 57:3389-3392. 10.2169/internalmedicine.1104-18

4. Walsh JP, Bremner AP, Bulsara MK, et al.: Subclinical thyroid dysfunction as a risk factor for cardiovascular disease. Arch Intern Med. 2005, 165:2467-2472. 10.1001/archinte.165.21.2467

5. Erem C: Blood coagulation, fibrinolytic activity and lipid profile in subclinical thyroid disease: subclinical hyperthyroidism increases plasma factor X activity. Clin Endocrinol (Oxf). 2006, 64:323-329. 10.1111/j.13652265.2006.02464.x 
6. Cortese B, Berti S, Biondi-Zoccai G, et al.: Drug-coated balloon treatment of coronary artery disease: a position paper of the Italian Society of Interventional Cardiology. Catheter Cardiovasc Interv. 2014, 83:427435. 10.1002/ccd.25149

7. Moses JW, Leon MB, Popma JJ, et al.: Sirolimus-eluting stents versus standard stents in patients with stenosis in a native coronary artery. N Engl J Med. 2003, 349:1315-1323. 10.1056/NEJMoa035071

8. Lee DH, de la Torre Hernandez JM: The newest generation of drug-eluting stents and beyond . Eur Cardiol. 2018, 13:54-59. 10.15420/ecr.2018:8:2

9. Cassese S, Byrne RA, Tada T, et al.: Incidence and predictors of restenosis after coronary stenting in 10004 patients with surveillance angiography. Heart. 2014, 100:153-159. 10.1136/heartjnl-2013-304933

10. Dhakam S, Jafferani A, Ahmed H, Rahman N, Gowani A: Safety and efficacy of drug-eluting balloons in the treatment of drug-eluting in-stent restenosis: experience of a tertiary care hospital. J Invasive Cardiol. 2012, 24:335-338.

11. Shahabuddin S, Sami SA, Ansari JA, et al.: Coronary artery bypass grafting after percutaneous coronary intervention. J Coll Physicians Surg Pak. 2012, 22:340-341.

12. Beyer C, Plank F, Friedrich G, Wildauer M, Feuchtner G: Effects of hyperthyroidism on coronary artery disease: a computed tomography angiography study. Can J Cardiol. 2017, 33:1327-1334.

10.1016/j.cjca.2017.07.002

13. Canpolat U, Turak O, Özcan F, et al.: Impact of free thyroxine levels and other clinical factors on bare metal stent restenosis. Arch Endocrinol Metab. 2017, 61:130-136. 10.1590/2359-3997000000197

14. Qureshi IA, Khabaz MN, Baig M, Begum B, Abdelrehaman AS, Hussain MB: Histopathological findings in goiter: a review of 624 thyroidectomies. Neuro Endocrinol Lett. 2015, 36:48-52.

15. Lampridis S, Lau MC, Mhandu P, Parissis H: Concomitant off-pump coronary artery bypass grafting and total thyroidectomy for a large retrosternal goitre: a case report and review of the literature. J Thorac Dis. 2016, 8:E362-E368. 10.21037/jtd.2016.03.33 